KLEINE TEXTE FÜR THEOLOGISCHE UTND PHILOLOGISCHE VORLESUNGEN UND ÜBUNGEN

HERAUSGEGEBEN VON HANS LIETZMANN

58

\title{
ALTJÜDISCHE LITURGISCHE GEBETE
}

AUSGEWÄHLT UND MIT EINLEITUNGEN

HERAUSGEGEBEN

voN

PROF. D. W. STAERK

BONN

A. MARCUS UND E. WEBER'S VERLAG 
